\title{
Correlaciones entre el test de velocidad en 4 metros y el test de caminata en 6 min en enfermos respiratorios crónicos
}

\author{
PATRICIO RIOSECO S.*, MAYA RUBILAR V.*, LORENA ADRIAZOLA S.* y DANILO GÓMEZ C.**
}

\section{Correlations between the 4 meter speed test and the 6-minute walking test in chronic respiratory patients}

4-Meter Gait Speed Test (4MGST) a frailty test, is considered a surrogate for the 6-Minute Walk Test (6MWT) in COPD. However, it has not been investigated in other respiratory conditions. Over a year, we studied patients attending our Unit for evaluation with 6MWT, measuring the speed they achieved walking $4 \mathrm{~m}, 2 \mathrm{~h}$ before performing 6MWT. 162 patients (99 women) were studied; series' mean values were: age 65 years-old; body weight, $73 \mathrm{~kg}$; height, $158 \mathrm{~cm}$ and BMI, $29.4 \mathrm{~kg} / \mathrm{m}^{2} .36 \%$ of them had idiopathic pulmonary fibrosis, 17\% GOLD IV COPD, 11\% GOLD III COPD, $12 \%$ pulmonary arterial hypertension, $12 \%$ obstructive apnea-hypopnea syndrome, and 12\% other conditions. ANOVA showed no difference between diagnostic categories for both test. Average score in 6MWT was 368.5 $m$ and $1.01 \mathrm{~m} / \mathrm{s}$ in $4 M G S T$. Pearson correlation coefficient revealed significant positive correlation between results of both tests: high score in 4MGST is equivalent to high score in 6MWT. There was negative correlation with age and positive correlation with height. At one year follow-up, 16 patients had died. They obtained significantly lower scores in both tests (4MGST: $0.69 \mathrm{~m} / \mathrm{s}$ and 6MWT: $248.1 \mathrm{~m}$ ) Survival chance fell to $20 \%$ for patients walking slower than $0.69 \mathrm{~m} / \mathrm{s}$. It is possible to replace TC6M with T4M in patients with different respiratory pathologies, we can predict death for any cause if a subject walked at $\leq$ speed at $0.69 \mathrm{~m} / \mathrm{s}$. T4M is cheap and easy to perform in primary care, serving as a risk assessment to refer to a more complex center.

Key words: Walk test; Walking speed; Gait; Primary Health Care; Pulmonary Disease.

\section{Resumen}

La prueba de velocidad de marcha en 4 metros (T4M) es considerada sustituto de la caminata en 6 min (TC6M) en EPOC. Sin embargo, no ha sido bien investigada en otras enfermedades respiratorias. Durante un año estudiamos pacientes que concurrieron a nuestra Unidad para realizar el TC6M midiendo la velocidad alcanzada en 4 metros, 2 h previo a realizar el TC6M. De 162 pacientes 99 eran mujeres. La edad media fue de 65 años, peso de $73 \mathrm{~kg}$, talla de $158 \mathrm{~cm}, I M C 29,4 \mathrm{~kg} / \mathrm{m}^{2} .36 \%$ tenían fibrosis pulmonar idiopática, 17\% EPOC, GOLD IV,11\% EPOC, GOLD III, 12\% apnea de sueño y 12\% otras enfermedades. No hubo diferencia para los distintos diagnósticos en ambos tests. La distancia media en TC6M fue 368,5 m la velocidad: 1,01 m/s en T4M.Hubo una correlación positiva significativa entre ambos test: alto rendimiento en T4M es equivalente a un alto rendimiento en el TC6M. Hubo correlación negativa con la edad y positiva con la estatura. Al año de seguimiento 16 pacientes habían fallecido, siendo estos los que habian obtenido los más bajos rendimientos en ambos tests (T4M: $0,69 \mathrm{~m} / \mathrm{s}$ y 248,1 m en TC6M) La posibilidad de sobrevida cayó a $20 \%$ en aquellos individuos que alcanzaron una velocidad inferior a $0,69 \mathrm{~m} / \mathrm{s}$. Es posible sustituir el TC6M por el T4M en pacientes con diferentes patologías respiratoria, podemos predecir la muerte por cualquier causa si un sujeto camina a una velocidad $\leq 0,69 \mathrm{~m} / \mathrm{s}$, T4M es barato y fácil de realizar en atención primaria, sirviendo como evaluación de riesgo para referir a un centro más complejo.

Palabras Clave: Test de caminata; Velocidad de marcha; Atención de salud primaria; Enfermedad respiratoria.

\footnotetext{
* Unidad de Enfermedades Respiratorias, Hospital Las Higueras, Talcahuano. Talcahuano, Chile.

** M.Sc. Campus Concepción, Universidad de Concepción. Concepción, Chile.
} 


\section{Introducción}

Las pruebas funcionales respiratorias evalúan intercambio gaseoso; mecánica; capacidad de ejercicio y control respiratorio para obtener un diagnóstico, evaluar respuestas a tratamientos, definir riesgo quirúrgico y establecer pronóstico. Aquellas de capacidad de ejercicio ilustran la respuesta integral al estrés físico y psicológico. Aptitud muscular y capacidad funcional son factores pronósticos relacionados con calidad de vida y morbimortalidad en portadores de patología respiratoria. Medir la capacidad de ejercicio es esencial en la valoración inicial.

Las pruebas de ejercicio más utilizadas son el TC6M; la prueba de resistencia de caminata (ESWT) y la prueba incremental de resistencia a la caminata (ISWT). Menos conocidas son las pruebas de velocidad alcanzada como el T4M.

El TC6M sirve para seguimiento, respuesta al tratamiento y pronóstico en enfermedad pulmonar obstructiva crónica (EPOC), Hipertensión Arterial Pulmonar (HAP) y Fibrosis Pulmonar Idiopática (FPI), ubicación en lista de espera de trasplante pulmonar ${ }^{1} \mathrm{y}$ falla cardíaca ${ }^{2}$. Se ha utilizado para medir respuesta a esteroides inhalatorios en $\mathrm{EPOC}^{3}$ demostrando confiabilidad a corto y largo plazo evaluando progresión de la enfermedad, cambios en actividad física y predicción de sobrevida ${ }^{4,5,6}$.

El T4M mide fragilidad integrando: capacidad de ejercicio, reserva funcional pulmonar, calidad de vida, cociente metabólico, índice de masa corporal, edad y estado psicológico. Correlaciona bien con resultados mediocres en personas año$\operatorname{sas}^{7,8}$, con capacidad de ejercicio, grado de disnea medido por el cuestionario del Medical Research Council (mMRC) y calidad de vida medida por el cuestionario de Saint George en individuos con $\mathrm{EPOC}^{9}$. Menos evidencia de esta correlación existe en pacientes con fibrosis pulmonar idiopática $(\mathrm{FPI})^{10}$ y casi nada en otras entidades.

E1 TC6M es un test de ejercicio sub-máximo; mide la distancia alcanzada caminando rápidamente en una superficie plana controlando frecuencia cardíaca, presión arterial, \% de saturación de $0_{2}\left(\mathrm{Sa}_{2}\right)$ y disnea, durante $6 \mathrm{~min}$. Son normales distancias iguales o superiores a 350 $\operatorname{metros}^{11,12}$. La medición de la velocidad alcanzada caminando una distancia de 4 metros por su parte, se relaciona con la capacidad funcional y la mortalidad por cualquier causa en personas mayores considerándose normal velocidades iguales o superiores a $0,8 \mathrm{~m} / \mathrm{s}^{13}$. Es fácil y simple de realizar. No requiere gran espacio físico o equipamiento. Mide exclusivamente la velocidad alcanzada en 4 metros y solo se requiere de un cronómetro para llevarla a cabo.

Las pruebas tradicionales de ejercicio demandan tiempo, personal entrenado y algunas, equipos sofisticados. Necesitamos métodos simples para evaluar la declinación funcional en el día a día de la práctica clínica para utilizar de manera más provechosa nuestros siempre escasos recursos. Decidimos entonces evaluar si existe correlación entre el T4M y el TC6M en pacientes con enfermedades respiratorias no consideradas previamente, estudiar su confiabilidad y considerar su importancia como herramienta de evaluación pronostica en niveles Primarios de Salud.

\section{Material y Métodos}

Durante 24 meses, adultos, capaces de caminar y comprender instrucciones referidos por primera vez a nuestra Unidad para ser evaluados con TC6M fueron sometidos prospectivamente al siguiente protocolo:

1. Delimitamos una parte del corredor interno de nuestra Unidad, usando cinta adhesiva roja marcando un primer punto a 2 metros del comienzo del corredor de prueba y un segundo, 4 metros más adelante para tener espacios de aceleración y desaceleración de 2 metros cada uno. Explicamos al paciente el objetivo del test, debiendo comenzar a caminar a paso normal al escuchar: ¡COMIENCE! Un paramédico utilizando un cronómetro, registraba el momento en que el primer pie del paciente cruzaba el primer punto y luego, cuando el pie del paciente terminaba de cruzar el segundo punto. Se calculó los segundos utilizados para cubrir los 4 metros delimitados. Permitimos utilizar aparatos ortopédicos u $\mathrm{O}_{2}$ suplementario si el paciente era usuario de aquellos (Figura 1).

2. Dos horas después, los pacientes fueron sometidos al TC6M según las Guías de la American Thoracic Society:ATS/European Respiratory Society:ERS.

3. Exploramos la variabilidad de la velocidad registrándola en tres períodos separados de tiempo durante el TC6M: 1 a 2 min; 3 a 4 min y 5 a 6 min.

4. Calculamos el tiempo ocupado en realizar el $\mathrm{T} 4 \mathrm{M}$, seleccionando al azar un grupo de 10 pacientes registrando el tiempo desde la instrucción hasta la finalización del test.

5. Los pacientes fueron seguidos con protocolos habituales de nuestra Unidad, según diagnóstico basal registrando su estado vital durante el siguiente año a la realización del test. 


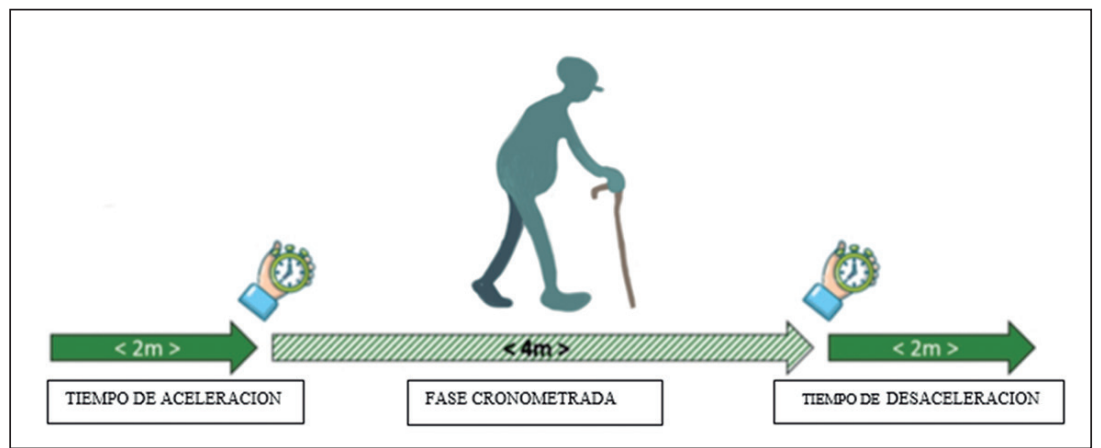

Figura 1. Representación esquemática del test de velocidad de la marcha en 4 metros (T4M).
6. Todos firmaron el formulario de Consentimiento Informado para pruebas de Ejercicio del Hospital La Higueras. El estudio fue aprobado por el Comité Ético-Científico de la Universidad de Concepción.

El criterio de exclusión al ingreso correspondió al del TC6M (ATS/ERS Guidelines) No excluimos pacientes durante las pruebas.

\section{Análisis estadísticos}

Presentamos los resultados de la correlación entre el TC6M y el T4M en relación a cinco patologías respiratorias obtenido del análisis descriptivo, utilizando técnicas estadísticas univariadas y multivariadas, comparación de grupos, y basados en las relaciones entre variables. Utilizamos el programa R-Project versión 3.2.2.

Para el análisis estadístico de correlaciones, utilizamos el test de correlación de Pearson. Para estudiar diferencias medias utilizamos la t de student para muestras independientes, para diferencias de medias múltiples el análisis de varianza (ANOVA), para el estudio de supervivencia el análisis Weibull y para evidenciar variables significativas en la probabilidad de muerte la regresión logística. El valor de significancia estadística fue de $\alpha=0,05$; todos los valores de " $p$ " inferiores a ese valor representan significancia.

\section{Resultados}

Estudiamos 162 pacientes, $91(56,2 \%)$ mujeres. 16 enfermos $(9,9 \%)$ habían fallecido luego de un año de seguimiento.

La edad media del grupo fue 65 años; el índice de masa corporal medio fue $29,4 \mathrm{~kg} / \mathrm{m}^{2}$. La distancia media alcanzada en TC6M fue 368,5 metros $(78,7 \%$ del predeterminado) y la velocidad media alcanzada en 4 metros fue $1,02 \mathrm{~m} / \mathrm{s}$.

La mayor proporción de pacientes tenía FPI: 36\%; seguidos por EPOC GOLD IV: 17\%; Síndrome de Apnea Hipopnea Obstructiva del Sueño (SAHOS): 12\%; Hipertensión Arterial Pulmonar (HAP): 12\%; Miscelánea:12\% y pacientes EPOC GOLD III: 11\% (Tabla 1).

La velocidad en tres períodos del TC6M no mostró variabilidad. En el intervalo de 1 a $2 \mathrm{~min}$ los sujetos alcanzaron una velocidad de $1,036 \mathrm{~m} / \mathrm{s}$ ( $\pm 0,315 \mathrm{DS})$; en el intervalo de 2 a $3 \mathrm{~min}, 1,011$ $\mathrm{m} / \mathrm{s}( \pm 0,340 \mathrm{DS})$ y en el intervalo de 5 a $6 \mathrm{~min}$, $0,987 \mathrm{~m} / \mathrm{s}( \pm 0,360 \mathrm{DS})$. ANOVA reveló homo-

Tabla 1. Características demográficas y resultados del TC6M y del T4M en 162 pacientes respiratorios crónicos (91 mujeres y 71 hombres)

\begin{tabular}{|c|c|c|c|c|c|c|}
\hline Varial & & & Media & \multicolumn{3}{|c|}{ Cuartil } \\
\hline Edad & & & 65 & \multicolumn{3}{|c|}{$59-73$} \\
\hline Peso c & (kg) & & 73 & \multicolumn{3}{|c|}{$60-85$} \\
\hline Talla & & & 158 & \multicolumn{3}{|c|}{$152-166$} \\
\hline IMC ( & & & 29,4 & \multicolumn{3}{|c|}{$24,1-34$} \\
\hline TC6M & & & 368 & \multicolumn{3}{|c|}{$300,8-419,5$} \\
\hline $\mathrm{T} 4 \mathrm{M}(1$ & & & 1,01 & \multicolumn{3}{|c|}{$0,82-1,23$} \\
\hline Diagnóstico & FPI & EPOC IV & EPOC III & HAP & SAHOS & Otros \\
\hline Porcentaje \% & 36 & 17 & 11 & 12 & 12 & 12 \\
\hline
\end{tabular}

FPI: Fibrosis pulmonar idiopática; EPOC IV y III: Enfermedad pulmonar obstructiva GOLD IV y III; HAP: Hipertensión arterial pulmonar; SAHOS: Síndrome de apnea hipopnea obstructiva del sueño. 


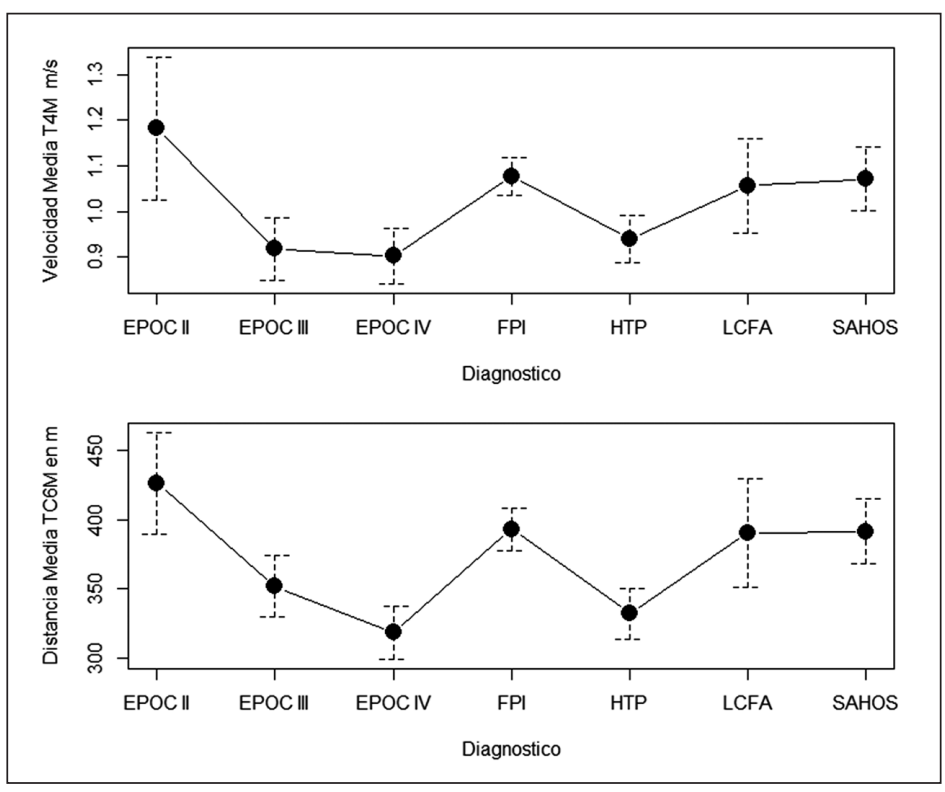

Figura 2. Gráficos de medias y desviaciones estándar de los valores de T4M y TC6M para pacientes con diferentes diagnósticos de enfermedades respiratorias. (EPOC II-II y IV según GOLD, FPI: fibrosis pulmonar idiopática; HTP: hipertensión arterial pulmonar; LCFA: Limitación crónica al flujo aéreo. Agregar: SAHOS: Síndrome de apnea hipopnea obstructiva del sueño. geneidad entre las categorías diagnósticas en términos de TC6M y T4M, p =0,127 $(\alpha>0,05)$ (el diagnóstico no predice resultados de TC6M o T4M). La Figura 2 muestra las medias del resultado en cada prueba e intervalo de confianza según diagnóstico.

Analizando variables antropométricas, encontramos correlación negativa significativa entre resultados del T4M y edad: altos valores en este test se asociaron a menor edad y viceversa $(\mathrm{p}=0,0001)$; correlación positiva significativa con la talla: individuos más altos caminaron más rápido los 4 metros $(\mathrm{p}=0,0007)$ y no encontramos correlación entre los resultados del test con el peso corporal $(\mathrm{p}=0,1554)$.

El coeficiente de correlación de Pearson para el grupo, mostró correlación significativa entre resultados del T4M y la distancia en metros alcanzada en el TC6M, igual que en el porcentaje del valor predictivo del test para cada paciente $(\mathrm{r}=0,92$, $\mathrm{p}<0,0001$ y $\mathrm{r}=0,79, \mathrm{p}<0,0001$ respectivamente). Altos valores en el T4M se asocian a altos valores en el TC6M y viceversa. Hay equivalencia entre ambos tests a este respecto (Figura 3).

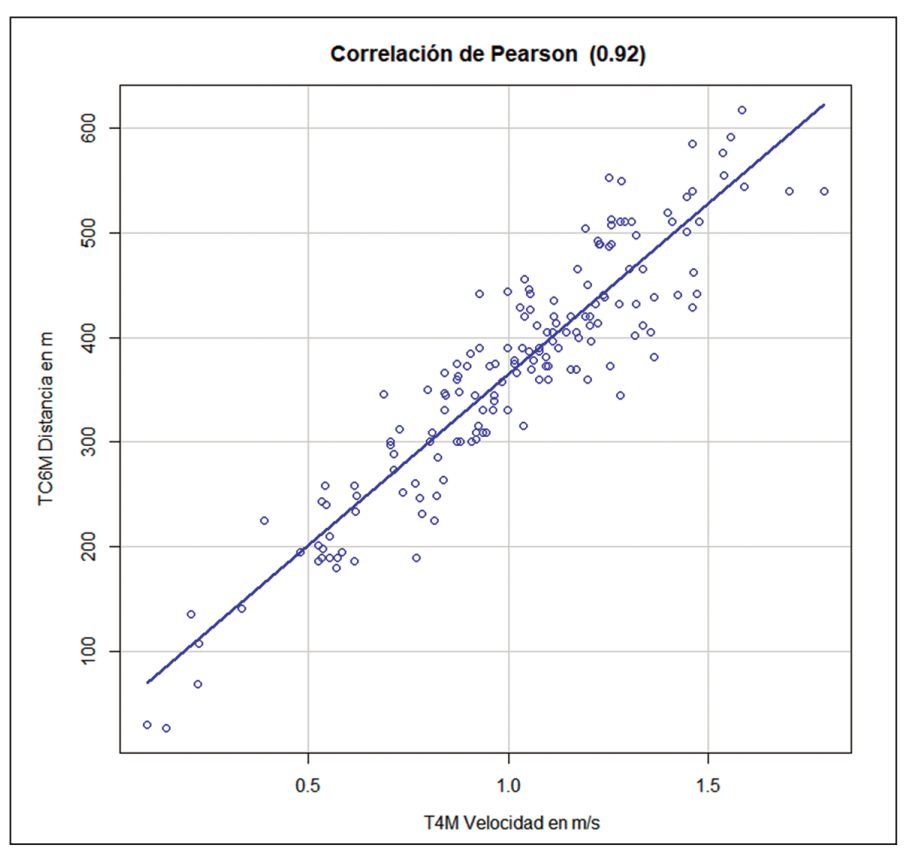

Figura 3. Correlación entre ambos tests: distancia recorrida en caminata de $6 \mathrm{~min}$ (TC6M) y velocidad de la marcha en $4 \min (\mathrm{T} 4 \mathrm{M})$ en todos pacientes evaluados. Aplicando el test de correlación de Pearson se obtuvo un valor de $r=0,92(p=0,0001)$. 
El análisis de subgrupos, según diagnósticos más frecuentes también demostró una correlación significativa entre los resultados de ambas pruebas. Para EPOC GOLD IV $\mathrm{r}=0,90$; para HAP $r=0,95$; para FPI $r=0,92$; y para SAHOS $\mathrm{r}=0,88$ (Figura $4 \mathrm{a}, \mathrm{b}, \mathrm{c}, \mathrm{d})$.

Comparando los resultados obtenidos entre los 16 pacientes fallecidos durante el año de seguimiento con los 146 sobrevivientes, usando el t-test encontramos diferencia significativa en la velocidad alcanzada en el T4M. Los que sobrevivieron al año, caminaron con una velocidad media de $1,04 \mathrm{~m} / \mathrm{s}$ versus $0,69 \mathrm{~m} / \mathrm{s}$ los fallecidos $(\mathrm{p}=0,00048)$. Igualmente hubo una diferencia significativa en la distancia caminada en 6 min; los sobrevivientes alcanzaron una distancia media de $381,71 \mathrm{~m}$ mientras que los fallecidos, caminaron una distancia media de $248,18 \mathrm{~m}$ $(p=0,0004)$. Ambos tests son equivalentes también en este aspecto (Figura 5).

Los diagnósticos con mayor mortalidad al año fueron: FPI y EPOC GOLD IV (43,7\% de los fallecidos), seguidos por EPOC GOLD III y HAP $(6,25 \%$ de los fallecidos respectivamente), consistente con la proporción de pacientes admitidos en cada categoría diagnóstica. La edad media de los fallecidos fue de 71,3 años (60-81) significativamente diferente a la del grupo completo. La edad media entre los sujetos fallecidos con diagnósticos más frecuentes no mostró diferen-

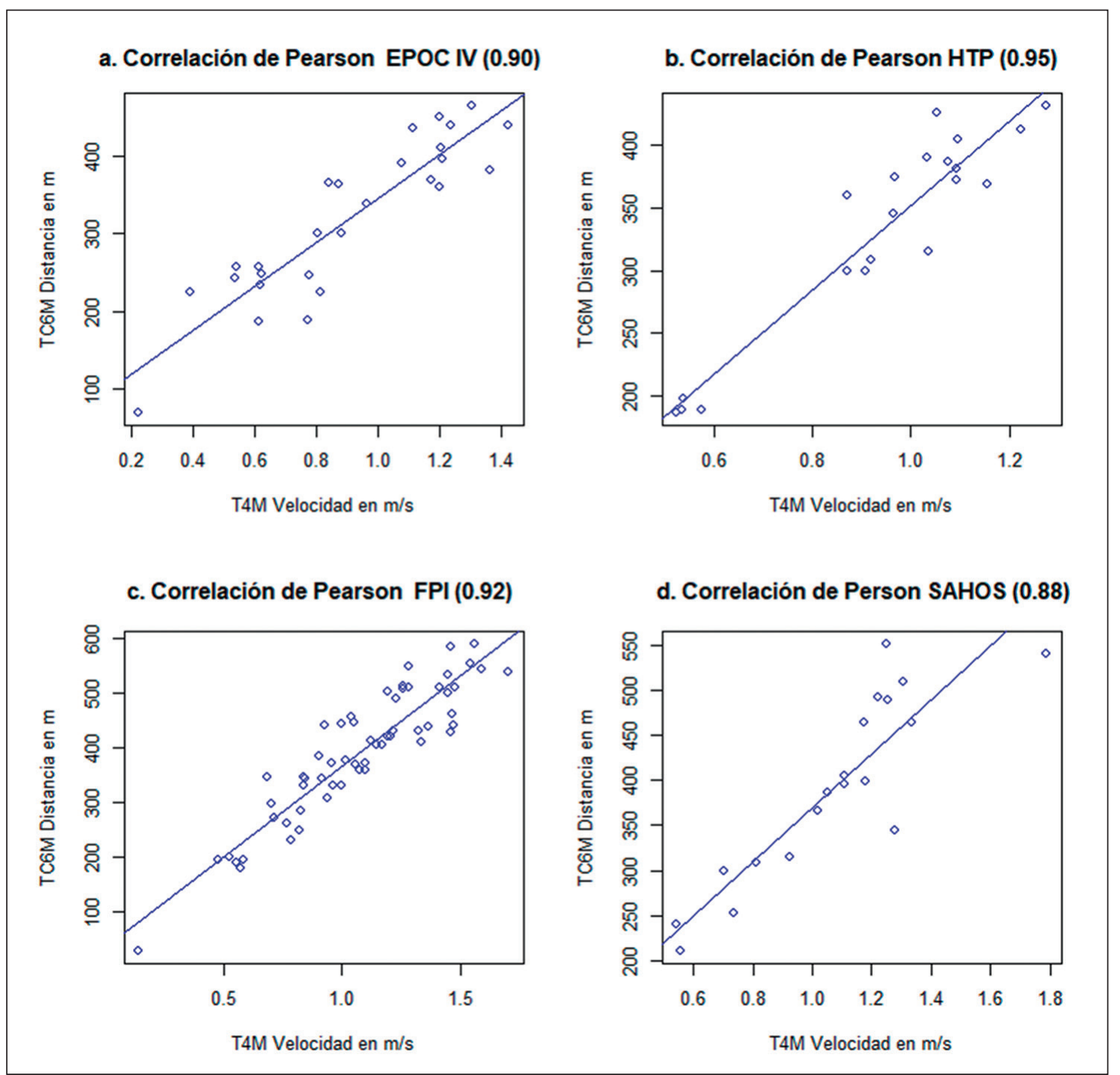

Figura 4. Correlación entre ambos tests: distancia recorrida en caminata de 6 min (TC6M) y velocidad de la marcha en 4 min (T4M) en pacientes agrupados según diagnóstico de su patología respiratoria: a) Enfermedad Pulmonar Obstructiva Crónica etapa IV GOLD $(\mathrm{n}=17, \mathrm{r}=0,90)$; b) Hipertensión arterial pulmonar $(\mathrm{n}=12, \mathrm{r}=0,95)$; c) Fibrosis pulmonar idiopática $(\mathrm{n}=36 ; \mathrm{r}=0,92)$; d) Síndrome Apnea Hipopnea Obstructiva del sueño $(\mathrm{n}=12, \mathrm{r}=0,88)$. 


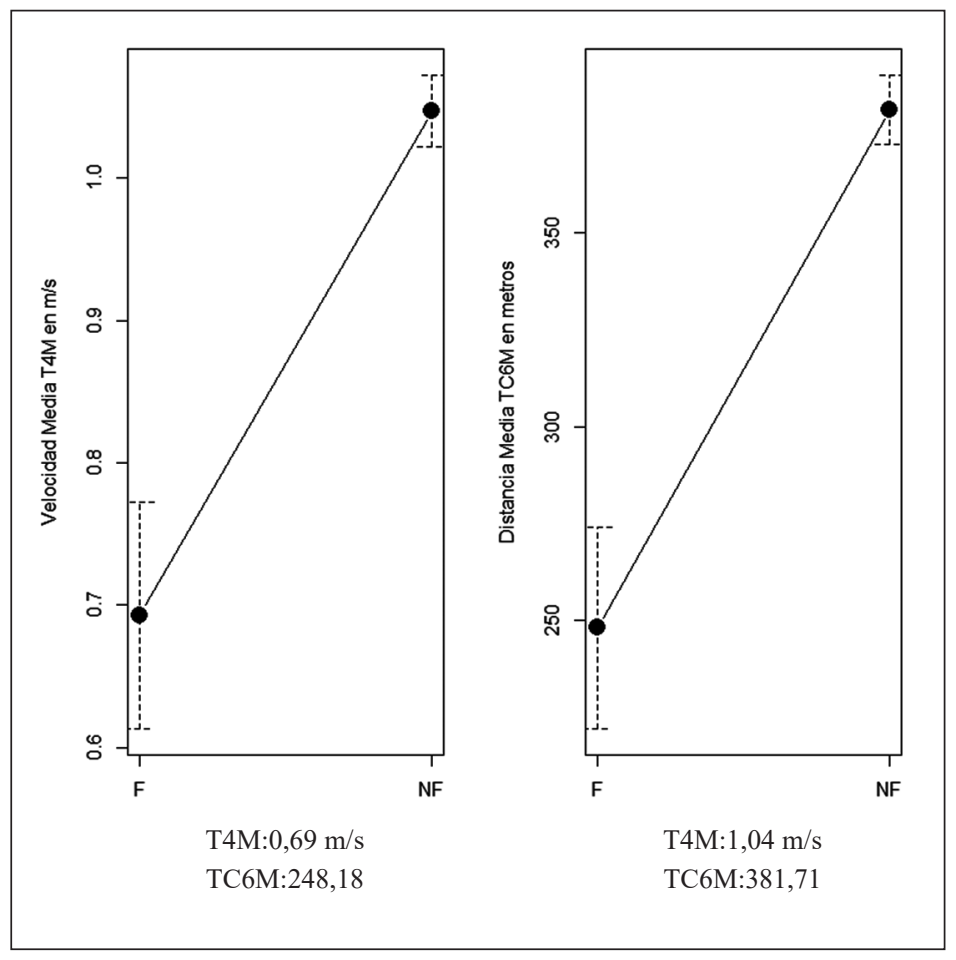

Figura 5. Gráficos de medias y DS de los valores de T4M y TC6M de 162 pacientes respiratorios para comparar los valores de las medias y DS de pacientes fallecidos $(\mathrm{F} ; \mathrm{n}=16)$ con los sobrevivientes $(\mathrm{NF} ; \mathrm{n}=146)$ al cabo de 1 año de seguimiento. F: Pacientes fallecidos al año de seguimiento. NF: Pacientes sobrevivientes cias (70,3 años para FPI y 72,8 años para EPOC GOLD IV)

El tiempo promedio utilizado en realizar el test en el grupo seleccionado de 10 pacientes fue de $182 \pm 8$ segundos (DS).

El análisis de Weibull predice la probabilidad de sobrevida en función del tiempo, desde la fecha en que se realiza el test. Después de 359 días de efectuada la prueba, para aquellos individuos que alcanzan una velocidad igual o inferior a 0,69 $\mathrm{m} / \mathrm{s}$, la probabilidad de sobrevivir cae a un $20 \%$ (Figura 6). La regresión logística muestra que caminar más lento, disminuye la probabilidad de mantenerse con vida en ese lapso (Figura 7).

\section{Discusión}

El TC6M, ESWT e IESWT constituyen métodos para evaluar capacidad de ejercicio, respuesta al tratamiento, pronóstico y tamizaje de pacientes con enfermedades respiratorias crónicas. La ATS y ERS publican guías para estandarizar su prácti$\mathrm{ca}^{11,12}$. Los tres son confiables en los procesos de test y retest, no hay diferencias de rendimiento entre patologías respiratorias diversas pero existe un efecto aprendizaje para el TC6M y el ISWT. En 1514 pacientes, Hernandes y colaboradores demostraron una mejoría media de $26,3 \mathrm{~m}$ (cuartiles: 24-29) en una segunda realización del TC6 $\mathrm{M}^{13,14}$.

El TC6M requiere un corredor sin obstácu- los de unos 30 metros de longitud, idealmente interior con temperatura confortable; aire acondicionado; ejecutor certificado en reanimación cardiopulmonar y conocedor del test. En el lugar debe haber facilidad para solicitar ayuda, administrar $0_{2}$ en caso necesario y una silla al final del corredor (fatiga del paciente). Se utiliza una escala de disnea validada (mMRC o la Escala de Borg) sensores de presión arterial, oxímetros de pulso y cronómetros. Debe disponerse de broncodilatadores y vasodilatadores coronarios (nitroglicerina sublingual), pues la mayoría de los examinados, posee reserva cardiorrespiratoria reducida. Debería haber un carro de paro cardíaco implementado disponible. No es necesaria la presencia del médico. Los requerimientos de personal idóneo y recursos físicos hacen difícil su realización diaria y frecuente.

La velocidad de marcha depende de la interrelación de funciones músculo-esqueléticas, visuales, neurológicas, de producción de energía y un estado estable cardiorrespiratorio ${ }^{15,16}$. $\mathrm{Su}$ disminución es signo de envejecimiento; se asocia a malas respuestas a rehabilitación y a enfermedades relacionadas con la edad ${ }^{17}$. Mide objetivamente funcionalidad en adultos mayores, se relaciona con declinación funcional corporal y con mortalidad ${ }^{18,19}$.

Las metodologías para efectuar test de caminata de campo no están estandarizadas; hay dis- 

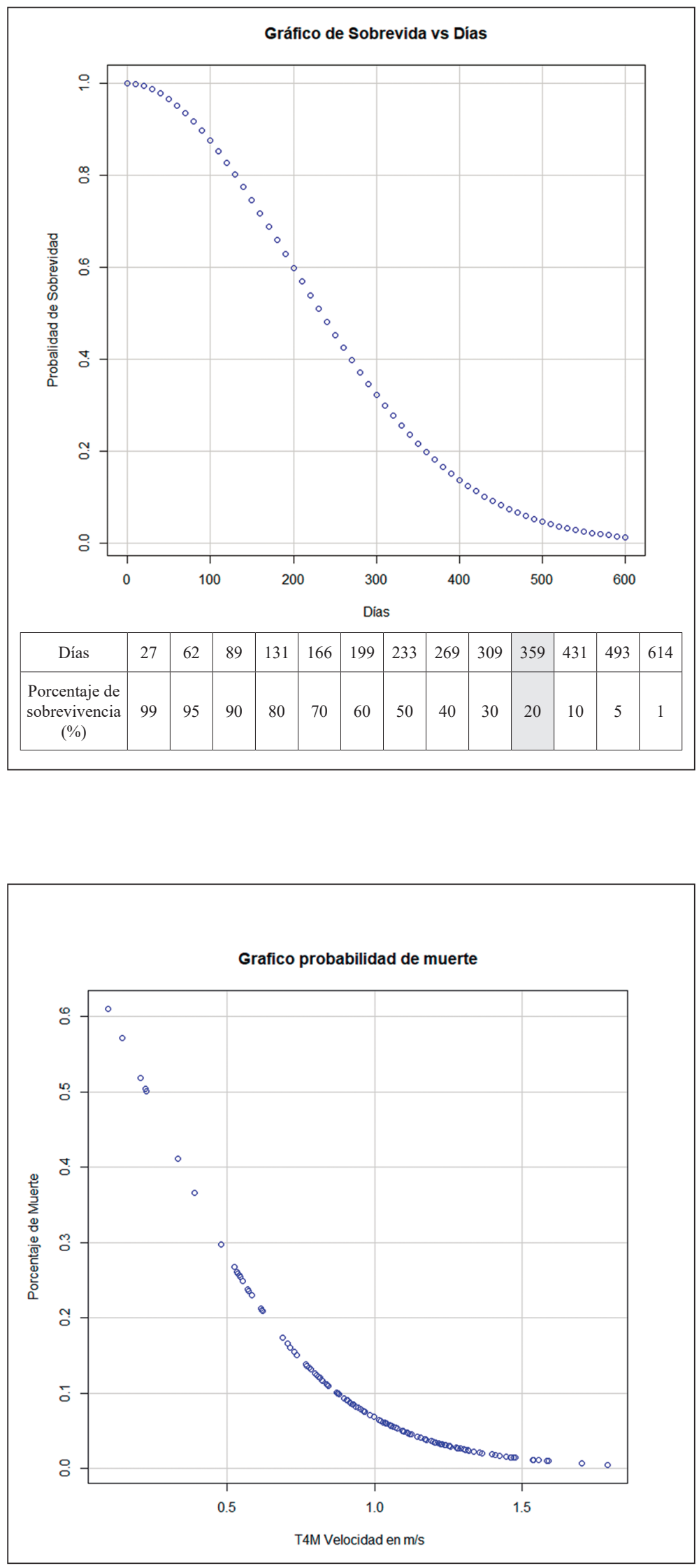

Figura 6. Probabilidad de sobrevida en función del tiempo transcurrido (días) después de la realización del T4M.
Figura 7. Probabilidad de muerte en función de la velocidad alcanzada en 4 min. 
cusión acerca de velocidad y distancia necesarias para obtener conclusiones válidas. Hee-Jae-Kim en 2016 concluyó que la medición de la velocidad de marcha a paso normal era adecuada para estimar funcionamiento físico y deterioro por enfermedades crónicas ${ }^{20}$. Analizó los resultados obtenidos por pacientes caminando a paso usual o rápido distancias de 4, 6 o 10 metros, observando que la reproducibilidad de la velocidad de marcha a paso normal fue mayor que a paso rápido. La distancia no tuvo efectos significativos en los resultados y el test y retest tuvieron excelente confiabilidad. Fernández-Huerta, en 136 personas mayores efectuando test de velocidad de marcha en 4 y 10 metros, demostró que había buena correlación entre ambas distancias y que el número de intentos no afectaba la velocidad ${ }^{21}$. Es probable entonces que para distancias cortas, no se produzca efecto aprendizaje ${ }^{22}$. Muñoz- Mendoza revisó sistemáticamente 69 estudios de test de caminata como medición de limitación funcional en mayores, apuntando al T4M como aquel que tiene los mejores resultados en fiabilidad intra paciente, interoperador e inter test ${ }^{23}$. Kon y colaboradores ${ }^{9}$ realizaron T4M en 80 pacientes EPOC por el mismo operador en dos oportunidades separadas 24 y $48 \mathrm{~h}$ obteniendo excelente confiabilidad con valores de Coeficiente de Correlación Intraclase (ICC) de 0,97 (95\% CI, 0,95-0,98) y 0,99 (95\% CI, 0,98-0,99) respectivamente. Los mismos autores, en 213 pacientes ingresados por exacerbación aguda de EPOC demostraron que el T4M realizado al alta es un predictor independiente de readmisión. Un tercio de los sujetos fue reingresado dentro de 90 días constituyendo una herramienta útil para planificar cuidados individualizados post alta ${ }^{24}$.

En clínica habitual la disponibilidad de espacio y tiempo limitan el cuidado óptimo de un gran número de pacientes. Sería valiosa una prueba simple, fácil y no consumidora de tiempo para el screening inicial. El T4M cumple esos requisitos por lo que decidimos comparar sus resultados con los del TC6M en pacientes de la vida real, independiente del diagnóstico basal.

Incluimos aquellos diagnósticos que más frecuentemente requieren evaluación especializada. La mayoría de los estudios realizados para validar el T4M ha sido en pacientes con EPOC. Encontramos escasa literatura acerca de su valor en otras condiciones respiratorias. Hirabayashi et al. examinaron 50 pacientes con enfermedad intersticial pulmonar la mayoría $(68,6 \%)$ FPI y encontraron una correlación significativa entre el T4M y el TC6M $(r=0,57$ y $\dot{\rho}<0,001)$ y buena correlación con la escala de disnea $\mathrm{mMRC}^{10}$. En- tre nuestros 162 pacientes, tuvimos 58 pacientes con FPI confirmada $(56,9 \%)$ obteniendo mejor correlación que la mencionada entre ambos exámenes.

Con respecto a diagnósticos y demografía, coincidimos con De Pew et al., quienes en 70 pacientes respiratorios crónicos reportan como diagnósticos más frecuentes: $\operatorname{EPOC}(51,4 \%)$; seguido por enfermedad intersticial pulmonar $(38,6 \%)$; HAP $(7,1 \%)$ y otros ${ }^{25}$. En nuestro grupo con más del doble de casos FPI constituyó el 36\% seguida por EPOC, con un 28\% constituyendo ambas condiciones patológicas la mayoría de los diagnósticos. Ambos estudios fueron similares en términos de edad media: 61,4 años en De Pew y 65 años en nuestra muestra, IMC media: 28 vs $29,4 \mathrm{~kg} / \mathrm{m}^{2}$ y sexo: mujeres $53 \%$ vs $56 \%$.

Respecto a resultados generales de pruebas de caminata, nuestros resultados coinciden con Fritz y colaboradores, quienes se refieren al T4M como al "sexto signo vital" encontrando que hay exceso de mortalidad por cualquier causa en los "caminadores lentos": pacientes que caminan a una velocidad inferior a $0,8 \mathrm{~m} / \mathrm{s}^{17}$. Studensky y colaboradores corroboran la correlación velocidad de marcha/sobrevida en adultos mayores ${ }^{26}$.

Ilgin et al. reportan que en EPOC, la velocidad de marcha disminuye al progresar la enfermedad. En pacientes EPOC IV la velocidad de marcha media, medida durante el TC6M fue de 1,0 $\pm 0,3$ $\mathrm{m} / \mathrm{s}^{27}$, comparable con nuestros resultados pues nuestros pacientes EPOC IV alcanzaron una velocidad media de $0,98 \pm 2 \mathrm{~m} / \mathrm{s}$ caminando 4 metros.

$\mathrm{Du}$ Bois et al. establecieron que caminar menos de 250 metros durante 6 min era factor independiente de mortalidad en pacientes con $\mathrm{FPI}^{28}$ y Nolan et al., fenotipificando las características asociadas a pacientes portadores de FPI encontraron que $37 \%$ de su población caminaba a menos de $0,8 \mathrm{~m} / \mathrm{s}$ en 4 metros lo que se relacionaba a edad mayor y a mayor IMC ${ }^{29}$.

En nuestros pacientes sobre $20 \%$ fueron "caminadores lentos" y el grupo total tenía sobrepeso. Aun cuando no hubo correlación fuerte entre los resultados del T4M e IMC en nuestros pacientes, encontramos también una correlación negativa significativa con la edad (edad media 71,36 años). Los pacientes con velocidad de marcha igual o superior a $1,01 \mathrm{~m} / \mathrm{s}$ eran significativamente más jóvenes (edad media 65 años). Los pacientes que al año de seguimiento habían fallecido, alcanzaron una velocidad igual o inferior a $0,69 \mathrm{~m} / \mathrm{s}$ versus los sobrevivientes que caminaron 4 metros a una velocidad igual o superior a 1,04 $\mathrm{m} / \mathrm{s}$. Igualmente, nuestros resultados en el TC6M se corresponden con los de Du Bois et al. pues los 
fallecidos al año de seguimiento, alcanzaron una distancia media de 248, 18 metros mientras los sobrevivientes, caminaron 381,71 metros en 6 min.

Analizando variabilidad en la velocidad de marcha, igual que en otros estudios ${ }^{25,30}$ no encontramos diferencias significativas al medir la velocidad en tres etapas diferentes del TC6M corroborando su estabilidad sugiriendo que un test de corta distancia provee información similar en algunos aspectos a test de mayor duración. Karpman et al., al examinar la factibilidad de implementar el T4M en 70 enfermos EPOC, demostró que el tiempo agregado a una visita clínica habitual era de $95 \pm 20$ segundos $^{31}$. En nuestro caso, en el subgrupo de 10 enfermos seleccionados al azar demoramos $182 \pm 8$ segundos en realizar de la prueba, tiempo no excesivo agregado a la visita clínica.

Tuvimos la ventaja de haber trabajado con diagnósticos diversos, no limitados solo a una entidad. En esta diversidad de cuadros, obtuvimos una correlación casi directa entre resultados del T4M y el TC6M. A mayor velocidad de marcha en $4 \mathrm{~m}$, mayor distancia alcanzada caminando $6 \mathrm{~min}$.

El análisis de subgrupos muestra que nuestros resultados se compara con De Pew et al. en pacientes con EPOC y corroboran los hallazgos de Kon et al..$^{9,25}$ que demuestran una correlación significativa entre los resultados del T4M y el ISWT el que a su vez correlaciona muy bien con el TC6M.

Seguir a la población testeada por un año nos permitió definir que un nivel crítico para el pronóstico vital en estos enfermos es alcanzar una velocidad igual o inferior a $0,69 \mathrm{~m} / \mathrm{s}$ como ilustra la figura 6 donde observamos que a los 359 días posteriores al examen, solo el $20 \%$ de los sujetos que alcanzaron esa velocidad están vivos.

Consideramos como limitante, que el nuestro fue un ensayo unicéntrico con pequeño número de enfermos en cada grupo diagnóstico, pues es imposible analizar correlaciones entre el T4M y el TC6M por patología e identificar un grupo específico con mayor riesgo de mortalidad al seguimiento. Un estudio multicéntrico con número mayor de pacientes, respondería a la interrogante de si una determinada patología determina un mejor o peor rendimiento y por tanto, si el T4M es de mayor utilidad en una u otra condición clínica.

\section{Conclusiones}

Sería posible substituir el TC6M por el T4M de acuerdo a sus resultados, en algunos pacientes con enfermedades respiratorias crónicas.
Podríamos predecir la muerte por cualquier causa durante el año posterior a la realización del test en pacientes que alcanzan una velocidad igual o inferior a $0,6 \mathrm{~m} / \mathrm{s}$ al caminar una distancia de 4 metros.

El T4M ocupa poco tiempo, es fácil de implementar, barato, no requiere grandes espacios, instrumentos sofisticados, ni mayor experiencia del ejecutor y se podría realizar antes de la visita clínica agregado a los signos vitales.

Puede ser implementado en nivel primario como índice de riesgo y derivación temprana en pacientes respiratorios.

\section{Bibliografía}

1.- EGAN TM, MURRAY S, BUSTAMI RT, SHEARON TH, MCCULLOUGH KD, EDWARDS LB, et al. Development of a new allocation system in the United States. Am J Transplant. 2006; 5: 1212-27.

2.- OPASICH C, PINNA GD, MAZZA A, FEBO O, RICCARDI R, RICCARDI PG, et al. Six minute walking performance in patients with moderate to severe heart failure: Is it a useful indicator in clinical practice? Eur Heart J. 2001; 22: 488-96.

3.- PAGGIARO PL, DAHLE R, BAKRAN I, FRITH L, HOLLINGWORTH K, EFTHIMIOU J. Multicentre randomised placebo-controlled trial of fluticasone propionate in patients with chronic obstructive pulmonary disease. International COPD Study Group. Lancet. 1998; 35: 773-80.

4.- HOLlAND AE, Hill CJ, DOWMAN L, GLASPOLE I, GOH N, LEE AL, et al. Short- and long-term reliability of the 6-minute walk test in people with idiopathic pulmonary fibrosis. Respir Care. 2018; 63: 994-1001. DOI: https://doi.org/10.4187/respcare.05875.

5.- WASCHKI B, KIRSTEN AM, HOLZ O, MUELLER KC, SCHAPER M, SACK AL, et al. Disease progression and changes in physical activity in patients with chronic obstructive pulmonary disease. Am J Respir Crit Care Med. 2015; 192: 295-306.

6.- PINTO-PLATA VM, COTE C, CABRAL H, TAYLOR J, CELLI BR. The 6-min walk distance: change over time and value as a predictor of survival in severe COPD. Eur Respir J. 2004; 23: 28-33.

7.- KARPMAN C, BENZO R. Gait speed as a measure of functional status in COPD patients. Int J COPD 2014: 9; 1315-20.

8.- FRIED LP, TANGEN CM, WALSTON J, NEWMAN C, HIRSCH J, GOTTDIENER T, et al. Frailty in older adults: evidence for a phenotype. J Gerontol A Biol Sci Med Sci. 2001; 56: M146-156.

9.- KON SS, PATEL MS, CANAVAN JL, CLARK AL, JONES SE, NOLAN CM, et al. Reliability and validity of 4-metre gait speed in COPD. Eur Respir J. 2013; 42: $333-40$. 
10.- HIRABAYASHI R, TAKAHASHI Y, NAGATA K, MORIMOTO T, WAKATA K, NAKAGAWA A, et al. The validity and reliability of four-meter gait speed test for stable interstitial lung disease patients: the prospective study. J Thorac Dis 2020; 12: 1296-1304. Disponible en: http://dx.doi.org/10.21037/jtd.2020.02.57.

11.- HOLLAND AE, SPRUIT MA, TROOSTERS T, PUHAN MA, PEPIN V, SAEY D, et al. An official European Respiratory Society/American Thoracic Society technical standard: field walking tests in chronic respiratory disease. Eur Respir J. 2014; 44: 1428-46.

12.- AGARWALA P, SALZMAN SH. Six minute walk test: Clinical role, technique, coding and reimbursement. Chest 2020; 157: 603-11. Doi:org/10.1016/j. chest.2019.10.014.

13.- FERNÁNDEZ-HUERTA L, CÓRDOVA-LEÓN K. Reliability of two gait speed tests of different timed phases and equal non-timed phases in communitydwelling older persons. Medwave. 2019; 19 (3): e7611. doi:10.5867/medwave.2019.03.7611

14.- HERNANDES NA, WOUTERS EF, MEIJER K, ANNEGARN J, PITTA F, SPRUIT MA. Reproducibility of the 6 minute walking test in patients with COPD. Eur Respir J. 2011; 38: 261-7.

15.- SINGH SJ, PUHAN MA, ANDRIANOPOULOS V, HERNANDES N, MITCHELL KE, HILL CJ, et al. An official systematic review of the European Respiratory Society/American Thoracic Society: measurement properties of field walking tests in chronic respiratory disease. Eur Respir J. 2014; 44: 1447-78.

16.- FRITZ S, LUSARDI M. White Paper: "Walking Speed: the Sixth Vital Sign". J Geriatr Phys Ther. 2009; 32: 2-5.

17.- PEEL NM, ALAPATT LJ, JONES LV, HUBBARD RE. The association between gait speed and cognitive status in community dwelling older people: a systematic review and meta-analysis. J Gerontol A Biol Sci Med Sci. 2019; 74: 943-8.

18.- MONTERO-ODASSO M, SCHAPIRA M, SORIANO ER, VARELA M, KAPLAN R, CAMERA LA, et al. Gait velocity as a single predictor of adverse events in healthy seniors aged 75 years and older. J Gerontol A Biol Sci Med Sci. 2005; 60: 1304-9.

19.- STUDENSKI S, PERERA S, WALLACE D, CHANDLER JM, DUNCAN PW, ROONEY E, et al. Physical performance measures in the clinical setting. J Am Geriatr Soc. 2003; 51: 314-22.

20.- ILYOECK PARK H-J-K, LEE HJ, LEE O. The reliability and validity of gait speed with different walking pace and distances against general health, physical function and chronic disease in aged adults. J Exerc Nutrition Biochem. 2016; 20: 46-50.

21.- FERNANDEZ-HUERTA L, CÓRDOVA-LEÓN K. Reliability of two gait speed tests of different timed phases and equal non-timed phases in community-dwelling older person. Medwave. 2019; 19: e7611 doi: 10.5867/ medwave.2019.03.7611.

22.- PETERS DM, FRITZ SL, KROTISH DE. Assessing the reliability and validity of a shorter walk test compared with the 10-meter walk test for measurements of gait speed in healthy, older adults. Randomized controlled trial. J Geriatr Phys Ther. 2013; 36: 24-30. Doi: 10.1519/JPT.Ob013e318248e20d.

23.- MUÑOZ-MENDOZA CL, CABREO-GARCÍA J, REIG-FERRER A, CABANERO-MARTÍNEZ MJ. Evaluation of walking speed tests as measurement of functional limitations in elderly people: A structured review. Int J Clin Health Psychol. 2010; 10: 359-78.

24.- KON SS, JONES SE, SCHOFIELD S, BANYA W, DICKSON MJ, CANAVAN JL, et al. Gait speed and readmission following hospitalization for acute exacerbnation of COPD: a prospective study. Thorax. 2015; 70 : 1131-7. doi:10.1136/thoraxjnl-207046.

25.- DE PEW ZS, KARPMAN C, NOVOTNY P, BENZO RP. Correlations between gait speed, 6 minute walk distance, physical activity and self-efficacy in patients with severe chronic lung disease. Resp Care. 2013; 58 : 2-12 doi:10.4187/respcare.02471.

26.- SUTENSKI S, PERERA S, PATEL K, ROSANO C, FAULKNER K, INZITANI M, et al. Gait speed and survival in older adults. JAMA 2011; 305: 50-8.

27.- ILGIN D, OZALEVLI S, KILINC O, SEVIN C, CIMRI AH, UCAN ES, et al. Gait speed as functional capacity indicator in patients with chronic obstructive pulmonary disease. Ann Thorac Med. 2011; 6: 141-6.

28.- DU BOIS RM, ALBERA C, BRADFORD WZ, COSTABEL U, LEFF JA, NOBLE PW, et al. Six minute walk distance is an independent mortality predictor in patients with idiopathic pulmonary fibrosis. Eur Respir J. 2014; 43: 1421-9.

29.- NOLAN CM, MADDOCKS M, MAHER TM, CANAVAN JL, JONES SE, BARKER RE, et al. Phenotypic characteristics associated with slow gait speed in idiopathic pulmonary fibrosis. Respirology. 2018; 23 : 498-506 doi:10.1111/resp.13213.

30.- REYCHLER G, DEBATISSE M, LEBECQUE P, PIETERS T, LIISTRO G, GOHY S. Variability of gait speed during six minutes walking test in COPD and cystic fibrosis patients. Gait Posture 2016; 49: 36-40. doi:10.1016/j.gaitpost.2016.06.007.

31.- KARPMAN C, LEBRASSEUR NK, DEPEW Z, NOVOTNY P, BENZO R. Measuring gait speed in the outpatient clinic: Methodology and feasibility. Resp Care 2014; 59: 531-7.

Correspondencia a:

Dr. Patricio Rioseco Saavedra

Unidad de Enfermedades Respiratorias

Hospital Las Higueras.

Alto Horno 777. Talcahuano, Chile 4030000

Email: parioseco@gmail.com 